\title{
JAK Inhibition as a New Treatment Strategy for Patients with COVID-19
}

\author{
Farhad Seif ${ }^{a}$ b Hossein Aazami ${ }^{c}$ Majid Khoshmirsafa ${ }^{d}$ Monireh Kamali $^{\mathrm{e}}$ \\ Monireh Mohsenzadegan ${ }^{f}$ Majid Pornour ${ }^{g}$ Davood Mansouri ${ }^{\text {h-j }}$
}

${ }^{a}$ Department of Immunology and Allergy, Academic Center for Education, Culture, and Research, Tehran, Iran; ${ }^{b}$ Neuroscience Research Center, Iran University of Medical Sciences, Tehran, Iran; ' Endocrinology and Metabolism Research Center, Endocrinology and Metabolism Clinical Sciences Institute, Tehran University of Medical Sciences, Tehran, Iran; ${ }^{d}$ Department of Immunology, School of Medicine, Iran University of Medical Sciences, Tehran, Iran; e Rajaei Cardiovascular Medical and Research Center, Iran University of Medical Sciences, Tehran, Iran; ${ }^{\mathrm{f}}$ Department of Medical Laboratory Science, Faculty of Allied Medical Sciences, Iran University of Medical Sciences, Tehran, Iran; ${ }^{9}$ Department of Photo Healing and Regeneration, Medical Laser Research Center, Yara Institute, Academic Center for Education, Culture, and Research, Tehran, Iran; ${ }^{h}$ Department of Clinical Immunology and Infectious Diseases, National Research Institute of Tuberculosis and Lung Diseases, Shahid Beheshti University of Medical Sciences, Tehran, Iran; 'The Clinical Tuberculosis and Epidemiology Research Center, National Research Institute of

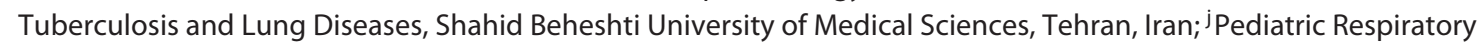
Diseases Research Center, National Research Institute of Tuberculosis and Lung Diseases, Shahid Beheshti University of Medical Sciences, Tehran, Iran

\section{Keywords}

Bricitinib · JAK-STAT pathway · JAK inhibitors .

Methotrexate $\cdot$ Cytokine $\cdot$ Angiotensin-converting enzyme inhibitor $\cdot$ Angiotensin receptor blocker

\begin{abstract}
After the advent of severe acute respiratory syndrome coronavirus 2 (SARS-CoV-2), the outbreak of coronavirus disease 2019 (COVID-19) commenced across the world. Understanding the Immunopathogenesis of COVID-19 is essential for interrupting viral infectivity and preventing aberrant immune responses before a vaccine can be developed. In this review, we provide the latest insights into the roles of angiotensinconverting enzyme II (ACE2) and Ang II receptor-1 (AT1-R) in
\end{abstract}

M.P. and D.M. contributed equally to this work as corresponding authors. Edited by: H.-U. Simon, Bern.

$\begin{aligned} & \text { karger@karger.com } \\ & \text { www.karger.com/iaa }\end{aligned}$
Karger $\%$

this disease. Novel therapeutic strategies, including recombinant ACE2, ACE inhibitors, AT1-R blockers, and Ang 1-7 peptides, may prevent or reduce viruses-induced pulmonary, cardiac, and renal injuries. However, more studies are needed to clarify the efficacy of these therapeutics. Furthermore, considering the common role of the Janus kinase-signal transducer and activator of transcription (JAK-STAT) pathway in AT1-R expressed on peripheral tissues and cytokine receptors on the surface of immune cells, potential targeting of this pathway using JAK inhibitors (JAKinibs) is suggested as a promising approach in patients with COVID-19 who are admitted to hospitals. In addition to antiviral therapy, potential ACE2- and AT1-R-inhibiting strategies, and other supportive care, we suggest other potential JAKinibs and novel anti-inflammatory combination therapies that affect 
the JAK-STAT pathway in patients with COVID-19. Since the combination of MTX and baricitinib leads to outstanding clinical outcomes, the addition of baricitinib to MTX might be a potential strategy.

(c) 2020 S. Karger AG, Basel

\section{Background}

After the sudden emergence of severe acute respiratory syndrome-coronavirus 2 (SARS-CoV-2) in Wuhan, China, in December 2019, coronavirus disease 2019 (COVID-19) conquered the world [1]. CoVs are a group of RNA viruses belonging to the Coronaviridae family. SARS-CoV-2 is detected within 1-2 days after the patient's symptoms, reaches a peak 4-6 days later, and is cleared within 18 days [2]. The clinical manifestations of COVID-19 range from asymptomatic to severe infections that require admission to an intensive care unit, with various mild to severe respiratory, gastrointestinal, hepatic, and neurologic involvements [3]. Such a novel virus with a high virulence and mortality necessitates further study to shed light on the immunopathogenesis of COVID-19 for the development of novel antiviral medications or vaccines to make the disease treatable or preventable, respectively.

\section{Angiotensin-Converting Enzyme II}

Recently, a critical host susceptibility factor, known as angiotensin-converting enzyme II (ACE2), has been identified for both SARS-CoV (SARS in 2003) and SARS-CoV-2 (COVID-19 in 2019) in humans and animals. It is crucial for entry into target cells with the help of the cellular protease TMPRSS2 $[4,5]$. The renin-angiotensin system is a pivotal regulator of blood pressure (Fig. 1) [6]. ACE2, which is highly expressed in the heart, lung, kidneys, brain, testes, liver, and small intestine [7], is a zinc carboxypeptidase and inactivates the potent vasoconstrictive peptide angiotensin II (Ang II) by removing the $\mathrm{C}$-terminal phenylalanine residue to yield heptapeptide Ang [1-7]. This conversion counterbalances the vasopressive function of Ang II [8]. Astonishingly, ACE2 binds to the trimers of spike proteins on the SARS-CoV-2 capsid, which triggers clathrin-dependent concomitant endocytosis of SARS-CoV-2 and ACE2 [9]. Following the interaction of the spike protein and ACE2, a disintegrin and metalloprotease 17 (ADAM17) is activated; consequently, it leads to shed- ding of ACE2 from the cell surface [10], thereby producing locally increased Ang II and hyaluronan (HA) levels resulting in the development of acute respiratory distress syndrome (ARDS) [11], which is the main cause of death in patients with COVID-19. Therapeutic interventions with soluble ACE2 or anti-ACE2 antibodies may impair the contribution of SARS-CoV-2 and ACE2.

Administration of soluble receptor-binding domain (RBD), the main domain derived from the SARS S-protein that can bind to the ACE2 and contains 193 amino acids, can effectively block the entry of SARS in cell culture. In addition, an antibody that binds to the ACE2 protein can be used to block this receptor. Nevertheless, several issues remain to be taken into consideration before applying these novel strategies, including the dose of administration to saturate ACE2-binding capacity owing to the ubiquitous ACE2 presence throughout the body, turnover or recycling of ACE2 on the surface of target cell, and local or systemic administration. Finally, binding both suggested ACE2 blockers may paradoxically improve or exacerbate the symptoms of the lung injury. Researchers suggest that it should be best administered during the initial phase of infection or as a prophylaxis to inhibit viral entry [12]. As mentioned earlier, recent studies have established that the lung is full of clear jelly-like liquid in COVID-19. Although the nature of the lucid jelly remains to be fully elucidated, HA is one of the components found in this fluid. Inflammatory cytokines such as interleukin (IL)-1 and tumor necrosis factor- $\alpha$ (TNF- $\alpha$ ) strongly induce HA-synthase-2 (HAS2) in the lung's endothelial and epithelial cells, as well as fibroblasts. Whenever breathing becomes difficult, hyaluronidase should be applied intratracheally; meanwhile, hymecromone (4-methylumbelliferone; 4-MU), an inhibitor of HAS2, may be administered to inhibit HAS2 [13].

\section{Angiotensin II Receptors}

Two subtypes of $G$ protein-coupled receptors exist through which Ang II exerts its action, i.e., the Ang II type 1 receptor (AT1-R) and Ang II type 2 receptor (AT2-R) (Fig. 1). Most of the cardiovascular/renal-related functions of Ang II are generally attributed to AT1-R. The expression of AT2-R is high in the developing fetus, while it is expressed at very low levels in the adult cardiovascular system. Angiotensin II receptor blockers (ARBs) (losartan, valsartan, telmisartan, and 


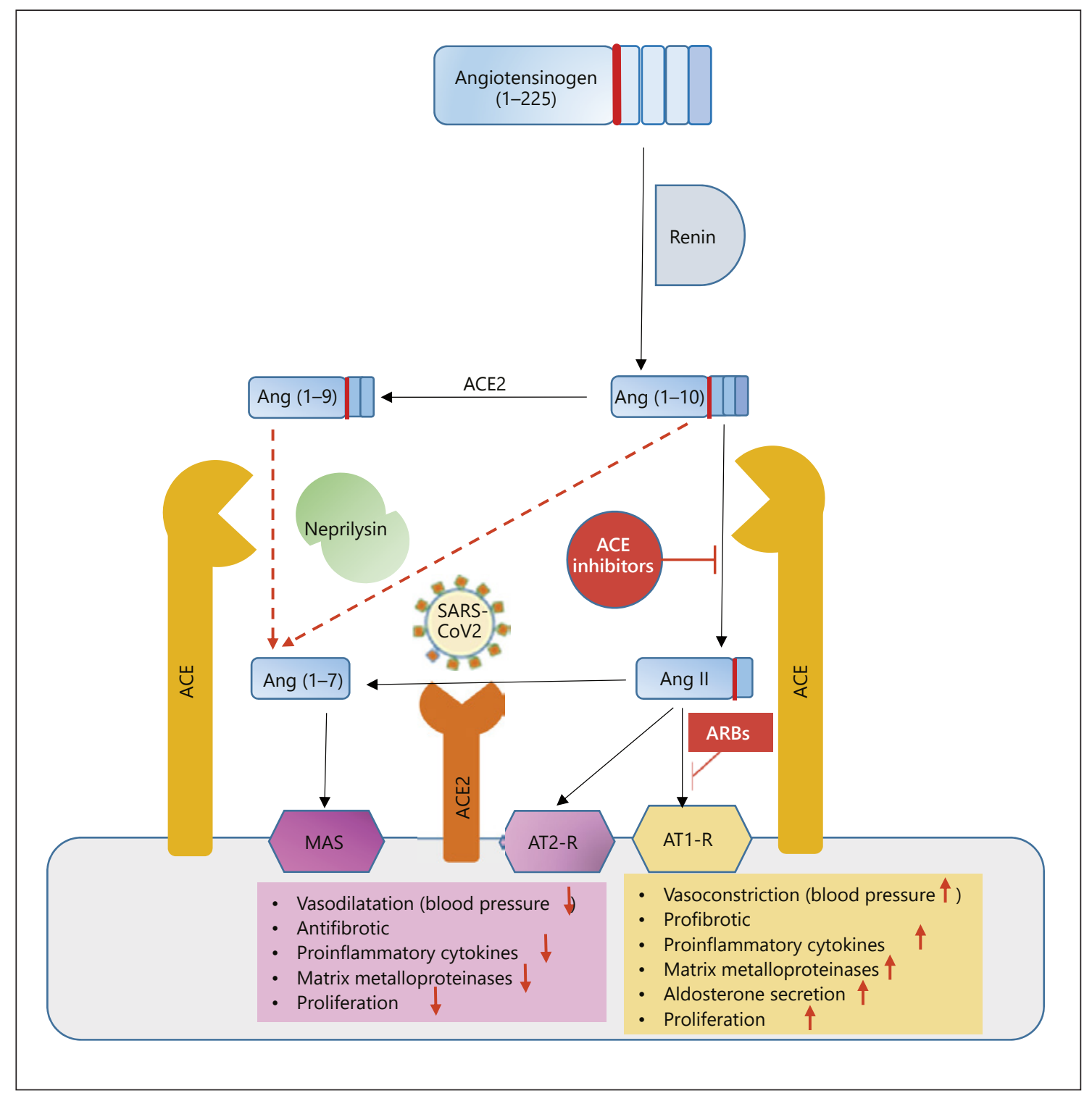

Fig. 1. The renin-angiotensin system is a pivotal regulator of hypertension, which controls the homeostasis of main electrolytes and body fluids. It starts with liver synthesis of the angiotensin (Ang) peptide precursor, named angiotensinogen. Subsequently, the potent vasoconstrictive peptide Ang II is cleaved by ACE, which increases blood pressure through the activation of Ang II type 1 (AT1R). In contrast, ACE2 inactivates Ang II by removing the C-terminal phenyl alanine residue to yield heptapeptide Ang (1-7), which exerts its opposing effects through the Mas receptor. Ang I is also cleaved to Ang (1-7) and Ang (1-9) by the action of neprilysin and ACE2, respectively. Then, Ang-1-9 is cleaved with the help of either NEP or ACE to produce Ang-1-7 in a minor pathway. Higher levels of Ang (1-7) originate from Ang II rather than Ang I and Ang (1-9). candesartan) are suggested for the treatment of COVID-19; however, contradictory outcomes have been reported so far. There are paradoxical and limited reports on ACE inhibitors (captopril and enalapril) and ARBs in patients with COVID-19 either to confirm or to prohibit the use of these therapeutics. Moreover, ACE2 may play beneficial rather than detrimental roles in patients with lung injury. In addition, a recent study indicated that elderly (age $>65$ years) patients with COVID-19 accompanied by hypertension comorbidity who are taking antihypertensive ARBs may be less likely to develop severe lung disease [14]. Innovative approach- 


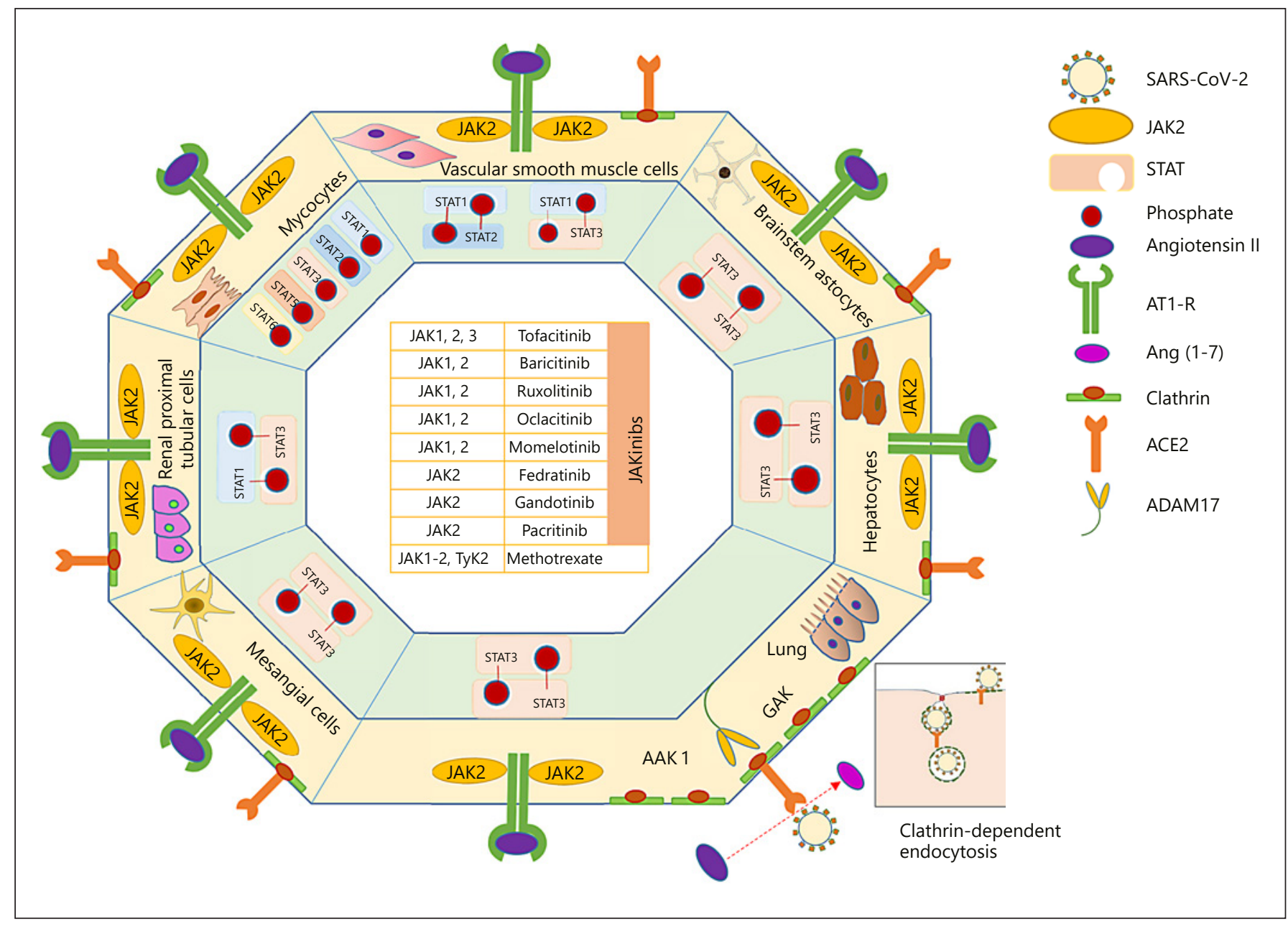

Fig. 2. Target cells potentially infected with SARS-CoV-2 in COVID-19.

es, such as recombinant ACE2, ACE inhibitors, ARBs, and Ang 1-7 peptides, as well as likely aldosterone synthase inhibitors, may prevent or reduce virus-induced pulmonary or cardiac damage. However, withdrawal of these inhibitors may be harmful in some high-risk patients with proven or suspected COVID-19. Consistent with the present guidelines, patients with hypertension or chronic kidney disease should keep taking antihypertensive drugs as administered by the physician without interruption because this action increases the readmission probability to hospitals and mortality rates in patients with heart and kidney failure $[15,16]$. Considering the equal efficacy but much lower side effects, ARBs may be used more favorably in patients who are at a higher risk of developing severe COVID-19.

\section{AT1-R and Cytokine Signaling through the Janus Kinase-Signal Transducer and Activator of Transcription Pathway in COVID-19}

Of note, Ang II mediates its actions through the Janus kinase-signal transducer and activator of transcription (JAK-STAT) signaling pathway, leading to vasoconstriction, hypertension, and chronic tissue injury [17]. The JAK-STAT pathway is involved in orchestrating the immune system to play a coordinated symphony. There are 4 mammalian proteins that belong to the nonreceptor JAK family, i.e., JAK1, JAK2, JAK3, and Tyk2. JAK1-2 and TYK2 are found ubiquitously, whereas JAK3 seems to act in hematopoietic lineage and exerts critical roles in lymphocyte function. In addition, human STAT family is comprised of 7 STAT, i.e., STAT1, STAT2, STAT3, 


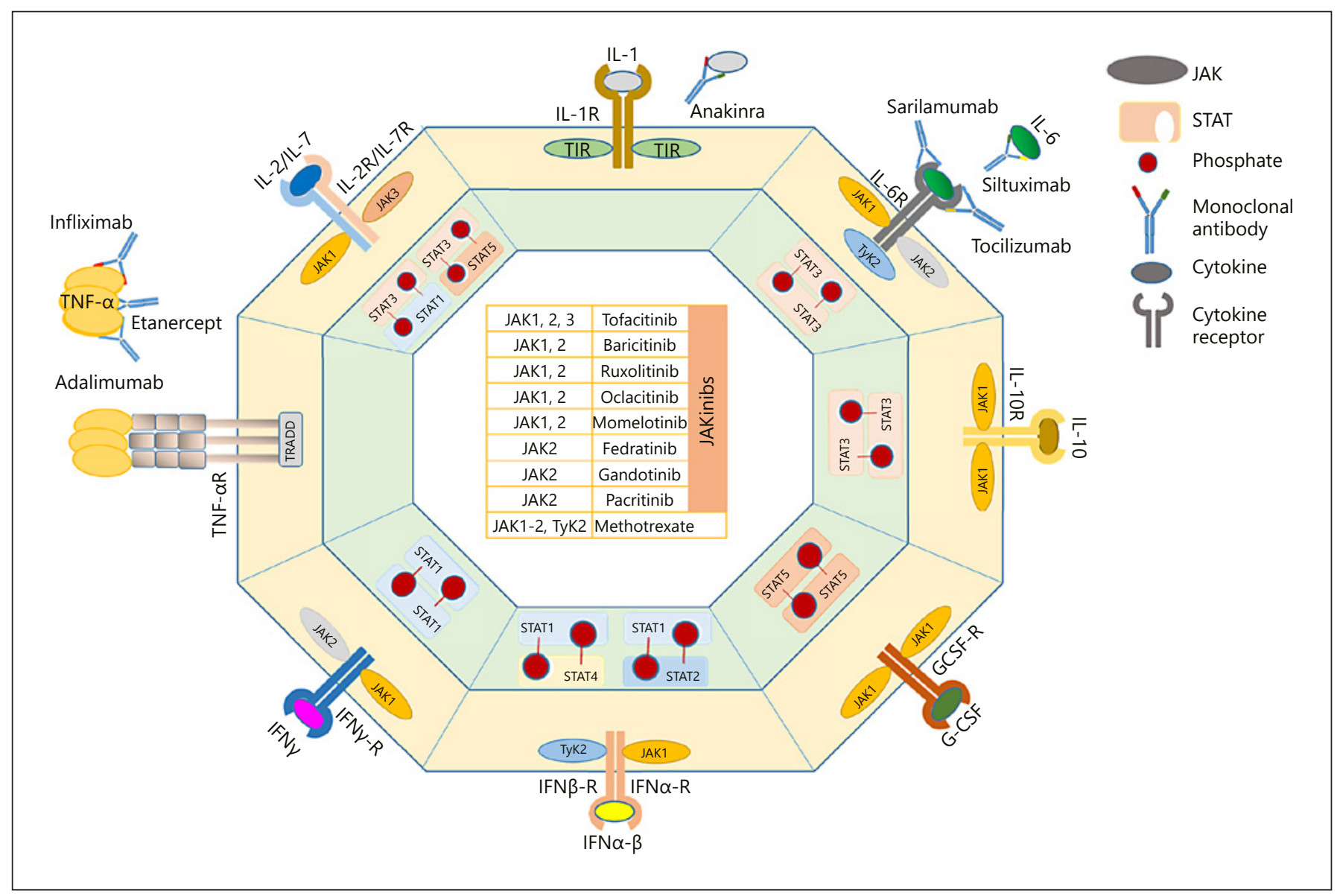

Fig. 3. Cytokines and cytokine receptors participating in the COVID-19 immunopathogenesis. G-CSF, granulocyte colony stimulating factor.

STAT4, STAT5A, STAT5B, and STAT6 [18]. The reninangiotensin system and proinflammatory cytokines have reciprocal relationships. Indeed, Ang II augments the infiltration of immune cells which, in turn, increases the local production of proinflammatory cytokines such as TNF- $\alpha$, IL-1, IL-6, and interferon (IFN)-y in target tissues [19]. Ang II signaling can be mediated through the JAKSTAT pathway in the cardiovascular system, renal proximal tubular cells, mesangial cells, brainstem astrocytes, and hepatocytes (Fig. 2). To this end, AT1-R recruits and phosphorylates JAK2 and, based on the target cell, one of the STAT is phosphorylated by activated JAK2. On the other hand, another mechanism suggested for a subgroup of patients with severe COVID-19 developing ARDS may be a cytokine storm, which is defined as the release of high concentrations of proinflammatory cytokines, including IL-1, IL-2, IL-6, IL-7, IL-10, TNF- $\alpha$ (Fig. 3), granulocytecolony stimulating factor, interferon (IFN)- $\gamma$ inducible protein-10 (IP-10), monocyte chemoattractant protein-1 (MCP-1), and macrophage inflammatory protein 1- $\alpha$ (MIP1- $\alpha$ ) by immune cells. The cytokine storm initiates a fulminant response by the immune system, leading to multiple organ failure and even death in severe COVID-19 [20]. Accordingly, since not only AT1-R but also a lot of cytokine receptors use this pathway for signal transduction, targeting the JAK-STAT pathway may provide a new avenue toward the management of COVID-19 before a vaccine can be developed.

\section{JAK Inhibitors}

JAK inhibitors (JAKinibs) are biologic agents that inhibit type I/II cytokine receptors. They are currently being used for the treatment of some diseases, and secondgeneration selective Jakinibs are being designed and in- 
vestigated [21]. Tofacitinib is an effective oral JAK2/1/3 inhibitor that was approved by the FDA for the treatment of rheumatoid arthritis (RA) in 2012 (Fig. 2, 3) [18]. Since JAK3 is limited to cytokines using the common $\gamma$ chain family, tofacitinib can effectively block IL-2, IL-7, and IL-6. However, herpes zoster and cellulitis infections may occur. Both low-density lipoprotein and high-density lipoprotein levels increase while blood neutrophils are decreased. In a study in patients with RA it decreased erythrocyte sedimentation rates and C-reactive protein levels [19]. Nevertheless, no treatment of COVID-19 with tofacitinib has been reported to date. Baricitinib is a selective JAK1/JAK2 inhibitor approved for the treatment of RA [18]. Both SARS-CoV-2 host cells (using AT1-R) and a large number of immune cells (using cytokine receptors) employ JAK2 for signal transduction through the JAK-STAT signaling pathway, and thus finding suitable and efficient JAKinibs or other immunosuppressive drugs may improve the treatment of COVID-19 (Fig. 2, 3). In this regard, the latest studies show that baricitinib is a potential treatment for ARDS in COVID-19. Furthermore, it can decrease the virus infectivity for lung cells. The ACE2 receptor has several regulators among which AP2-associated protein kinase-1 (AAK1) and cyclin G-associated kinase (GAK) mediate clathrin-dependent endocytosis (Fig. 2). Baricitinib not only interrupts the passage and intracellular assembly of SARS-CoV-2 into the target cells via disruption of AAK1 signaling but it also reduces the inflammation in patients with ARDS [22]. Although in a study in patients with RA who showed an inadequate response to prior methotrexate (MTX) treatment a dose-dependent decrease in hemoglobin levels was evident [23], a decrease in neutrophil counts together with an increase in low-density lipoprotein and high-density lipoprotein levels was observed in another study in patients with refractory RA following the use of baricitinib [24]. In contrast, tofacitinib does not significantly inhibit AAK1 [25]. Stebbing et al. [26] revealed that 3 JAKinibs, including baricitinib, ruxolitinib, and fedratinib, had similar JAK inhibitor potencies, while the high affinity of baricitinib to AAK1 and the merit of its once-daily oral dosing made it the best of these JAKinibs. Contrary to the other antiarthritic drugs or JAK inhibitors, baricitinib can inhibit clathrin-dependent endocytosis. Although JAK inhibition may weaken host inflammatory responses and impair hematopoiesis somehow, combination therapy using baricitinib and direct-acting antivirals, including lopinavir or ritonavir and remdesivir, can decrease viral infection and amplification, as well as unwanted host inflammatory reactions
[26]. Trials in human lupus have been initiated using baricitinib with a dose of $4 \mathrm{mg}$ daily, with improved clinical symptoms. Several trials, including phase 3 trials, have been completed for many disorders such as psoriasis, alopecia areata, allergic dermatitis, dermatomyositis, and graft-versus-host disease (GVHD) [27]. Other JAKinibs such as ruxolitinib, memolitinib, and oclacitinib target both JAK1 and JAK2 which can potentially affect signaling pathways downstream of the receptors involved in COVID-19 development (Fig. 2, 3). Fedratinib and pacritinib are other JAK2 inhibitors, and clinical trials are ongoing for the treatment of myelofibrosis. Pardanani et al. [28], who conducted a phase 1 trial in 59 patients with a high- or intermediate-risk of primary myelofibrosis using fedratinib, showed that this drug had a mild impact on serum levels of cytokines but more than half of the patients with myelofibrosis benefited from durable, rapid, and well-tolerated improvements in their clinical manifestations such as fatigue, night sweats, cough, and pruritus [28]. Gandotinib is another JAK2 inhibitor, and clinical trials are ongoing for the treatment of myeloproliferative disorders. The findings of a phase $1 / 2$ trial with 19 subjects indicated a reduction in spleen size in $22 \%$ of the patients suffering from myeloproliferative neoplasms. However, the main adverse effects were diarrhea, electrolyte imbalance, anemia, and nausea [29]. A phase 1 study of gandotinib in patients with primary myelofibrosis, polycythemia vera, and essential thrombocythemia showed acceptable safety and tolerability profiles [30]. They suggested that a phase 2 dose of $120 \mathrm{mg}$ was associated with clinical improvement [27]. A phase 2 study of Gandotinib in patients with myeloproliferative neoplasms established an effective response in patients previously treated with ruxolitinib. They reported no significant hematological, neurological, or infectious toxicities [31].

Using a JAKinib to treat a viral infection may play a double role because both type I IFN (IFN- $\alpha / \beta)$ and type II IFN (IFN-y) use the JAK-STAT signaling pathway. Cameron et al. [32] conducted a microarray study, which established that patients with SARS who were discharged from hospitals showed low IFN- $\alpha$ and IFN-y signaling while IFN- $\alpha$ and IFN-y signaling was evident in patients who died. Nevertheless, the use of pegylated IFN did not lead to beneficial antiviral outcomes and clinical trials with systemic administration of IFN- $\alpha / \beta$ resulted in conflicting findings. Animal model studies on MERS and SARS diseases demonstrated that IFN- $\alpha / \beta$ therapy may be beneficial in the early inflammatory phase of both diseases, whereas this therapy is harmful in the late phase of 
the diseases [33], suggesting that when hospital care is needed for patients with COVID-19, JAK inhibitors may be a promising approach. Nearly $80 \%$ of patients with COVID-19 can eradicate the virus, mostly via antiviral immune responses, especially IFN- $\alpha / \beta$. Thus, it is recommended that baricitinib or other JAK inhibitors be given to patients who are admitted to hospitals. Short-term use of baricitinib (i.e., 7-14 days) might reactivate no latent infections, including herpesviruses or tuberculosis, in these patients. Prospective studies (i.e., NCT04320277 and NCT04321993) are ongoing with baricitinib in these patients, which along with appropriate classification, definition of prognostic models, and evaluation of cytokine levels during the early and late phases of the disease, may provide new insights into the treatment of COVID-19 [34].

\section{JAKinib Monotherapy or Combination Therapy with MTX}

MTX is a folate antagonist that inhibits the activity of dihydrofolate reductase and the synthesis of purines. It is broadly used for the treatment of RA and psoriatic arthritis, which regresses proliferative cells in the $S$ phase. One of the main MTX mechanisms of action consists of targeting the JAK/STAT pathway [35], thereby downregulating the production of proinflammatory cytokines, i.e., IL-1, IL-12, and TNF- $\alpha$, while upregulating IL-4 and IL-10 as anti-inflammatory cytokines [36]. However, MTX monotherapy does not significantly affect IL-6 production [37]. Interestingly, Thomas et al. [34] in 2015 reported that MTX could inhibit JAK2 and JAK1 in Drosophila melanogaster and several human cell lines, e.g., Hodgkin lymphoma and acute myeloid leukemia, respectively. In silico modeling suggested that this inhibition may be due to direct binding of MTX to the JAK2 kinase domain ATP binding pocket [38]. The combination of MTX with JAK1 or JAK3 inhibitor leads to better clinical outcomes than monotherapy, while its combination with JAK1/JAK2 or JAK1-specific inhibitors does not seem to exert an additive clinical benefit. A phase 3 randomized controlled trial of IL-1 blockade (anakinra) in sepsis demonstrated a prominent survival benefit in hyperinflammatory conditions, without elevated adverse effects [34]. A multicenter, randomized controlled trial for the efficacy and safety of tocilizumab (anti-IL-6R) was recently approved in patients with COVID-19 pneumonia and high concentrations of IL-6 in China (ChiCTR2000029765) [39]. Considering mechanisms of action other than TNF- $\alpha$ inhibition (adalimumab or etanercept), only tocilizumab was established as obviously superior to MTX. Therefore, researchers believe that MTX has an efficacy equal to that of anti-TNFa monotherapy through adenosine and JAK inhibition. These effects possibly explain why MTX monotherapy results in good clinical outcomes resembling those of anti-TNF- $\alpha$ biologics alone but is less effective in comparison to anti-IL6-R alone [40]. MTX plus tofacitinib showed clinical effects similar to those of MTX plus adalimumab [41], suggesting that the specific inhibition of tofacitinib in combination with the JAK1/JAK2 inhibition capability of MTX may exert synergistic effects. There are conflicting data regarding the combination of baricitinib and MTX. A clinical trial reported that MTX plus baricitinib does not result in significantly better clinical outcomes, obtained by baricitinib alone [42]. In contrast, Fleischmann et al. [42] demonstrated that baricitinib monotherapy or combined with MTX had a higher efficacy and safety compared to MTX monotherapy as a treatment for patients with moderate to severe RA [42]. A more recent study showed that baricitinib was approved for either monotherapy or combination therapy with MTX in the treatment of moderate to severe RA [43]. Another study established that structural damage progression was less likely to occur with a combination of baricitinib and MTX rather than MTX or baricitinib alone [44]. Despite all of the possible benefits, JAK inhibition may decrease the host inflammatory response and MTX therapy may predispose the patient to zoster infection as a side effect. All current JAKinibs bind to highly similar catalytic ATP binding sites of JAKs and block them. Nevertheless, these kinds of inhibitors cannot discriminate infected target cells from healthy cells or mutated JAKs from wild-type ones, resulting in potential side effects. Therefore, specific inhibition and no interference with other JAKs remain to be clarified.

\section{Conclusion}

ACE2 and AT1-R play pivotal roles in COVID-19 development, and thus targeting of them using novel therapeutic strategies, including recombinant ACE2, ACE inhibitors, ARBs, and Ang 1-7 peptides, may prevent or decrease virus-induced pulmonary, cardiac, and renal damage. However, more studies are needed to clarify the efficacy of these therapeutics. On the other hand, JAKinibs may be beneficial because they may not only reduce the clinical symptoms in the multiple organs such as the 
lung, kidneys, and heart (because of blocking AT1-R) that are affected during the disease but also modulate some inflammatory cytokines (because of blocking the actions of type I/II cytokine receptors) that are released during ARDS or cytokine storm conditions. Therefore, JAKinibs are suggested as a promising approach in patients with COVID-19 who are admitted to hospitals. In this regard, it has been recommended that baricitinib or other JAK inhibitors might be given to patients with COVID-19 who are in the late inflammatory phase. Furthermore, there is controversy over combination therapy of baricitinib and MTX or either monotherapy, but according to recent studies the combination of MTX and baricitinib is suggested as a potential approach in the management of COVID-19. The modest safety, cost-effectiveness, and need for a low dose consumption of MTX make it an appealing alternative for combination therapy. Eventually, in addition to antiviral therapy, ACE2 inhibitors, ARBs, and other supportive care, we suggest other potential JAKinibs and novel anti-inflammatory combination therapies that affect JAK-STAT pathway in patients with COVID-19 to improve the treatment process and decease adverse effects because, although JAKinibs are highly effective, their high cost and adverse effects may limit their application. Therefore, low-cost alternative medications should be introduced to obviate the need for expensive and harmful treatments of $\mathrm{CO}$ VID-19.

\section{Availability of Data and Materials}

Please contact the corresponding author for data requests.

\section{Acknowledgment}

We would like to express our sincere gratitude to John J. O'Shea who taught us the JAK-STAT signaling pathway.

\section{Disclosure Statement}

The authors declare that they have no competing interests.

\section{Funding Source}

The authors received neither funding/support, nor grant for the publication of this article.

\section{Author Contributions}

Farhad Seif designed this study and drafted this paper. Hossein Aazami was involved in data collection. Majid Pornour and Majid Khoshmirsafa created the figures and interpreted the data. Farhad Seif, Monireh Kamali, and Monireh Mohsenzadegan critically revised this paper for important intellectual content. Davood Mansouri and Majid Pornour supervised this study. All of the authors read and approved the final version of this work.

\section{References}

1 Lai CC, Shih TP, Ko WC, Tang HJ, Hsueh PR. Severe acute respiratory syndrome coronavirus 2 (SARS-CoV-2) and coronavirus disease-2019 (COVID-19): the epidemic and the challenges. Int J Antimicrob Agents. 2020 Mar;55(3):105924.

2 Zou L, Ruan F, Huang M, Liang L, Huang H, Hong Z, et al. SARS-CoV-2 viral load in upper respiratory specimens of infected patients. $\mathrm{N}$ Engl J Med. 2020 Mar;382(12):1177-9.

3 Bai Y, Yao L, Wei T, Tian F, Jin DY, Chen L, et al. Presumed asymptomatic carrier transmission of COVID-19. JAMA. 2020 Feb; 323(14): 1406.

4 Zhou P, Yang XL, Wang XG, Hu B, Zhang L, Zhang W, et al. Discovery of a novel coronavirus associated with the recent pneumonia outbreak in humans and its potential bat origin. bioRxiv. 2020. doi: https://doi.org/ 10.1101/2020.01.22.914952.

5 Hoffmann M, Kleine-Weber H, Krüger N, Mueller MA, Drosten C, Pöhlmann S. The novel coronavirus 2019 (2019-nCoV) uses the SARS-coronavirus receptor ACE2 and the cellular protease TMPRSS2 for entry into tar- get cells. bioRxiv. 2020. doi: https://doi. org/10.1101/2020.01.31.929042.

6 Tan Z, Wu J, Ma H. Regulation of angiotensin-converting enzyme 2 and Mas receptor by Ang-(1-7) in heart and kidney of spontaneously hypertensive rats. J Renin Angiotensin Aldosterone Syst. 2011 Dec;12(4):413-9.

7 Tipnis SR, Hooper NM, Hyde R, Karran E, Christie G, Turner AJ. A human homolog of angiotensin-converting enzyme. Cloning and functional expression as a captopril-insensitive carboxypeptidase. J Biol Chem. 2000 Oct; 275(43):33238-43.

8 Clayton D, Hanchapola I, Thomas WG, Widdop RE, Smith AI, Perlmutter P, et al. Structural determinants for binding to angiotensin converting enzyme 2 (ACE2) and angiotensin receptors 1 and 2. Front Pharmacol. 2015 Jan; 6:5.

9 Inoue $Y$, Tanaka N, Tanaka Y, Inoue S, Morita K, Zhuang M, et al. Clathrin-dependent entry of severe acute respiratory syndrome coronavirus into target cells expressing ACE2 with the cytoplasmic tail deleted. J Virol. 2007 Aug; 81(16):8722-9.
$10 \mathrm{Xu}$ J, Sriramula S, Xia H, Moreno-Walton L, Culicchia F, Domenig O, et al. Clinical relevance and role of neuronal AT1 receptors in ADAM17-mediated ACE2 shedding in neurogenic hypertension. Circ Res. 2017 Jun; 121(1):43-55.

11 Hällgren R, Samuelsson T, Laurent TC, Modig J. Accumulation of hyaluronan (hyaluronic acid) in the lung in adult respiratory distress syndrome. Am Rev Respir Dis. 1989 Mar;139(3):682-7.

12 Kruse RL. Therapeutic strategies in an outbreak scenario to treat the novel coronavirus originating in Wuhan, China. F1000 Res. 2020 Jan;9:72.

13 Corman VM, Landt O, Kaiser M, Molenkamp R, Meijer A, Chu DK, et al. Detection of 2019 novel coronavirus (2019-nCoV) by real-time RT-PCR. Euro Surveill. 2020 Jan;25(3).

14 Liu Y, Huang F, Xu J, Yang P, Qin Y, Cao M, et al. Anti-hypertensive angiotensin II receptor blockers associated to mitigation of disease severity in elderly COVID-19 patients. medRxiv. 2020. doi: https://doi.org/10.1101/2 020.03.20.20039586. 
15 Vaduganathan M, Vardeny O, Michel T, McMurray JJ, Pfeffer MA, Solomon SD. ReninAngiotensin-Aldosterone System Inhibitors in Patients with Covid-19. N Engl J Med. 2020 Apr;382(17):1653-9.

16 Oliveros E, Oni ET, Shahzad A, Kluger AY, Lo $\mathrm{KB}$, Rangaswami J, et al. Benefits and risks of continuing angiotensin-converting enzyme inhibitors, angiotensin II receptor antagonists, and mineralocorticoid receptor antagonists during hospitalizations for acute heart failure. Cardiorenal Med. 2020;10(2):69-84.

17 Recinos A 3rd, LeJeune WS, Sun H, Lee CY, Tieu BC, Lu M, et al. Angiotensin II induces IL-6 expression and the Jak-STAT3 pathway in aortic adventitia of LDL receptor-deficient mice. Atherosclerosis. 2007 Sep;194(1):12533.

18 Seif F, Khoshmirsafa M, Aazami H, Mohsenzadegan M, Sedighi G, Bahar M. The role of JAK-STAT signaling pathway and its regulators in the fate of T helper cells. Cell Commun Signal. 2017 Jun;15(1):23.

19 Lee EB, Fleischmann R, Hall S, Wilkinson B, Bradley JD, Gruben D, et al.; ORAL Start Investigators. Tofacitinib versus methotrexate in rheumatoid arthritis. N Engl J Med. 2014 Jun;370(25):2377-86.

20 Huang C, Wang Y, Li X, Ren L, Zhao J, Hu Y, et al. Clinical features of patients infected with 2019 novel coronavirus in Wuhan, China. Lancet. 2020 Feb;395(10223):497-506

21 Schwartz DM, Bonelli M, Gadina M, O'Shea JJ. Type I/II cytokines, JAKs, and new strategies for treating autoimmune diseases. Nat Rev Rheumatol. 2016 Jan;12(1):25-36.

22 Richardson P, Griffin I, Tucker C, Smith D, Oechsle O, Phelan A, et al. Baricitinib as potential treatment for 2019-nCoV acute respiratory disease. Lancet. 2020 Feb;395 (10223):e30-1.

23 Keystone EC, Taylor PC, Drescher E, Schlichting DE, Beattie SD, Berclaz PY, et al. Safety and efficacy of baricitinib at 24 weeks in patients with rheumatoid arthritis who have had an inadequate response to methotrexate. Ann Rheum Dis. 2015 Feb;74(2):333-40.

24 Genovese MC, Kremer J, Zamani O, Ludivico C, Krogulec M, Xie L, et al. Baricitinib in patients with refractory rheumatoid arthritis. $\mathrm{N}$ Engl J Med. 2016 Mar;374(13):1243-52.

25 Sanchez GA, Reinhardt A, Ramsey S, Wittkowski H, Hashkes PJ, Berkun Y, et al. JAK1/2 inhibition with baricitinib in the treatment of autoinflammatory interferonopathies. J Clin Invest. 2018 Jul;128(7):3041-52.
26 Stebbing J, Phelan A, Griffin I, Tucker C, Oechsle O, Smith D, et al. COVID-19: combining antiviral and anti-inflammatory treatments. Lancet Infect Dis. 2020 Apr;20(4): 400-2.

27 Gadina M, Le MT, Schwartz DM, Silvennoinen $\mathrm{O}$, Nakayamada S, Yamaoka K, et al. Janus kinases to jakinibs: from basic insights to clinical practice. Rheumatology. 2019;58:i4-i16.

28 Pardanani A, Gotlib JR, Jamieson C, Cortes JE, Talpaz M, Stone RM, et al. Safety and efficacy of TG101348, a selective JAK2 inhibitor, in myelofibrosis. J Clin Oncol. 2011 Mar; 29(7):789-96.

29 Furqan M, Mukhi N, Lee B, Liu D. Dysregulation of JAK-STAT pathway in hematological malignancies and JAK inhibitors for clinical application. Biomark Res. 2013 Jan; 1(1):5.

30 Verstovsek S, Mesa RA, Salama ME, Li L, Pitou C, Nunes FP, et al. A phase 1 study of the Janus kinase 2 (JAK2)V617F inhibitor, gandotinib (LY2784544), in patients with primary myelofibrosis, polycythemia vera, and essential thrombocythemia. Leuk Res. 2017 Oct;61:89-95.

31 Berdeja J, Palandri F, Baer MR, Quick D, Kiladjian JJ, Martinelli G, et al. Phase 2 study of gandotinib (LY2784544) in patients with myeloproliferative neoplasms. Leuk Res. 2018 Aug;71:82-8.

32 Cameron MJ, Bermejo-Martin JF, Danesh A, Muller MP, Kelvin DJ. Human immunopathogenesis of severe acute respiratory syndrome (SARS). Virus Res. 2008 Apr;133(1): 13-9.

33 Channappanavar R, Fehr AR, Zheng J, Wohlford-Lenane C, Abrahante JE, Mack M, et al. IFN-I response timing relative to virus replication determines MERS coronavirus infection outcomes. J Clin Invest. 2019 Jul;130(9): 3625-39.

34 Favalli EG, Biggioggero M, Maioli G, Caporali R. Baricitinib for COVID-19: a suitable treatment? Lancet Infect Dis. 2020 Apr;S1473-3099(20)30262-0.

35 Thomas S, Fisher KH, Snowden JA, Danson SJ, Brown S, Zeidler MP. Methotrexate is a JAK/STAT pathway inhibitor. PLoS One. 2015 Jul;10(7):e0130078.

36 Friedman B, Cronstein B. Methotrexate mechanism in treatment of rheumatoid arthritis. Joint Bone Spine. 2019 May;86(3): 301-7.
37 Neurath MF, Hildner K, Becker C, Schlaak JF, Barbulescu K, Germann T, et al.; ZUM BÜSCHENFELDE HM. Methotrexate specifically modulates cytokine production by T cells and macrophages in murine collagen-induced arthritis (CIA): a mechanism for methotrexate-mediated immunosuppression. Clin Exp Immunol. 1999 Jan;115(1):42-55.

38 Palandri F, Labate C, Sabattini E, Catani L, Martino B. Low-dose methotrexate as treatment of myeloproliferative neoplasms: proof of principle of clinical activity. Am J Hematol. 2016 Aug;91(8):E329-30.

39 Mehta P, McAuley DF, Brown M, Sanchez E, Tattersall RS, Manson JJ; HLH Across Speciality Collaboration, UK. COVID-19: consider cytokine storm syndromes and immunosuppression. Lancet. 2020 Mar;395(10229): 1033-4.

40 Gremese E, Alivernini S, Tolusso B, Zeidler MP, Ferraccioli G. JAK inhibition by methotrexate (and csDMARDs) may explain clinical efficacy as monotherapy and combination therapy. J Leukoc Biol. 2019 Nov; 106(5): 1063-8.

41 Fleischmann R, Mysler E, Hall S, Kivitz AJ, Moots RJ, Luo Z, et al.; ORAL Strategy investigators. Efficacy and safety of tofacitinib monotherapy, tofacitinib with methotrexate, and adalimumab with methotrexate in patients with rheumatoid arthritis (ORAL Strategy): a phase $3 \mathrm{~b} / 4$, double-blind, head-tohead, randomised controlled trial. Lancet. 2017 Jul;390(10093):457-68.

42 Fleischmann R, Schiff M, van der Heijde D, Ramos-Remus C, Spindler A, Stanislav M, et al. Baricitinib, methotrexate, or combination in patients with rheumatoid arthritis and no or limited prior disease-modifying antirheumatic drug treatment. Arthritis Rheumatol. 2017 Mar;69(3):506-17.

43 Kawalec P, Śladowska K, Malinowska-Lipień I, Brzostek T, Kózka M. New alternative in the treatment of rheumatoid arthritis: clinical utility of baricitinib. Ther Clin Risk Manag. 2019 Feb;15:275-84.

44 Van der Heijde D, Durez P, Schett G, Naredo E, Østergaard M, Meszaros G, et al. Structural damage progression in patients with early rheumatoid arthritis treated with methotrexate, baricitinib, or baricitinib plus methotrexate based on clinical response in the phase 3 RA-BEGIN study. Clin Rheumatol. 2018 Sep; 37(9):2381-90. 